\title{
Coccygectomy as a Surgical Option in the Treatment of Chronic Traumatic Coccygodynia: A Single-Center Experience and Literature Review
}

\author{
Alexander Antoniadis ${ }^{1}$, Nils Harry-Bert Ulrich ${ }^{2}$, Hueseyin Senyurt ${ }^{1,3}$ \\ ${ }^{I}$ Department of Orthopedics, University Hospital Duesseldorf, University of Duesseldorf, Duesseldorf, Germany \\ ${ }^{2}$ Department of Neurosurgery and Neuroradiology, University Hospital Zurich, Zurich, Switzerland \\ ${ }^{3}$ Department of Orthopedic Surgery and Traumatology, Hospital Kemperhof-Koblenz, Koblenz, Germany
}

\begin{abstract}
Study Design: Retrospective cohort study.
Purpose: Trauma is the most common cause for chronic coccygodynia. The present study aims at presenting our results after complete removal of the coccyx for refractory traumatic coccygodynia in terms of pain level, complication rates, and patients' overall satisfaction.

Overview of Literature: There is limited extant literature describing the success rate and complications in refractory isolated traumatic coccygodynia.

Methods: From January 2011 to January 2012, 10 consecutive patients with posttraumatic coccygodynia (six males and four females; mean age, 42 years) were enrolled in our study. Conservative treatment of the condition had failed in all patients. The same surgeon performed a complete coccygectomy on all patients. Postoperative outcomes included measurements of pain relief and degree of patient satisfaction with the procedure's results.

Results: In our selected cohort, all patients indicated complete pain relief or significant pain improvement in follow up-care and would recommend this procedure. One patient developed a subcutaneous hematoma that required surgical intervention.

Conclusions: Our results suggest that complete removal of the coccyx relieves pain in patients with refractory chronic traumatic coccygodyniaand is therefore a reasonable treatment option after conservative treatment failure.
\end{abstract}

Keywords: Coccygectomy; Coccyx

\section{Introduction}

The word "coccyx" is derived from Greek; it refers to a cuckoo's beak, which resembles the anatomy of the curved coccygeal terminus [1]. Coccygodynia refers to a pathological condition in which pain occurs in the coccyx or its immediate vicinity [2]. The pain symptoms may be particularly acute when external force acts directly on the tailbone; this typically occurs during every-day activities such as sitting. Along with the bilateral ischialtuberosities, the coccyx bears the brunt of body weight during sitting. Notably, when a seated individual leans back, the weight load is borne almost entirely by the coccyx [3].

Coccygodynia typically affects adults [4], with a higher prevalence among women than men [5]. Trauma is the most common etiology of coccygodynia [6]. A fall onto

Received Mar 3, 2014; Revised Mar 31, 2014; Accepted Apr 11, 2014

Corresponding author: Hueseyin Senyurt

Department of Orthopedic Surgery and Traumatology, Hospital Kemperhof-Koblenz, Koblenz, Germany

Tel: +49261-4992236, Fax: +49261-4992237, E-mail: hueseyin.senyurt@gk.de 
one's buttocks may cause a contusion, fracture, or dislocation of the tailbone. Even such a minor, repetitive trauma such as sitting for long hours on hard or narrow surfaces (e.g., during cycling) can lead to chronic coccygodynia [7]. Degenerative changes that increase with age may lead to idiopathic coccygodynia [8]. Patients with coccygodynia typically complain of pain when sitting, especially while leaning back or standing up. Patients may also experience pain during defecation or sexual intercourse. Occasionally, the pain can radiate all the way to the pelvic floor and may mimic the symptoms of pelvic inflammatory disease. A digital rectal examination should be performed. The coccyx can be palpated between the thumb and forefinger, and is painful on palpation. Adjacent structures should be painless on examination. Completion of the diagnostic workup should include routine radiographic images of the coccyx in two planes. The magnetic resonance imaging (MRI) examination can be performed in selected cases, particularly to rule out a herniated disc or a tumorous process in the spine [9].

Initial treatment options for acute and chronic forms of coccygodynia include a range of conservative therapies. Adherence to pain medication, in conjunction with physiotherapeutic mobilization measures, can often times alleviate symptoms [8]. In addition, injections of local anesthetics or corticosteroids can be utilized. A surgical resection of the coccyx is performed only when complaints persist after all conservative measures have been exhausted. There are only a few studies in the literature that describe the clinical course and results following surgical excision of the coccyx for traumatic coccygodynia. There still remains substantive existing skepticism in performing this procedure. The aim of this study is to evaluate the success of complete removal of the coccyx for chronic traumatic coccygodynia and to compare our study results with the existing literature.

\section{Materials and Methods}

Between January 2011 and January 2012, ten consecutive patients who underwent surgical coccygectomy were enrolled in the study. The study included ten participants: six men and four women. The mean age of study participants was 41.9 years. All patients suffered from treatment-resistant coccygodynia and had exhausted conservative therapeutic options. All patients noted the regular intake of oral analgesics. The majority of patients (seven of ten) had already undergone infiltration treatment of the coccyx with a local anesthetic. Manual therapy techniques (five patients) and physical measures, such as the use of a seat ring (six patients), were frequently used. The preoperative duration of symptoms ranged from two to six years. The medical indication for surgical coccygectomy was recorded following consultation with the patient at our spinal surgery department's outpatient clinics. All patients reported a traumatic fall event onto the tailbone. There were no reported cases of idiopathic coccygodynia. Additionally, none of the patients had a reported psychiatric disorder.

On preoperative clinical examination, all patients showed marked tenderness on palpation over the coccyx. A digital rectal examination was performed to rule out a rectal-based pathology. All patients had undergone preoperative non-contrast radiographic imaging of the sacrum in two planes and an MRI scan of the region (Figs. 1, 2). The senior author (H.S.) performed surgery in all cases. All cases were performed in a prone position, and all patients also received perioperative antibiotic prophylaxis. A $5 \mathrm{~cm}$ midline skin incision was made approximately one $\mathrm{cm}$ above the gluteal cleft (Fig. 3). After dissection through the subcutaneous tissue, the fascia was opened and the coccyx was carefully removed using electrocautery (Fig. 4). Hemostasis was achieved using hemostatic agents (e.g., bone wax). In all patients, the entire coccyx was resected. Postoperatively, patients were allowed to move freely with full weight-bearing movement as tolerated by their pain level.

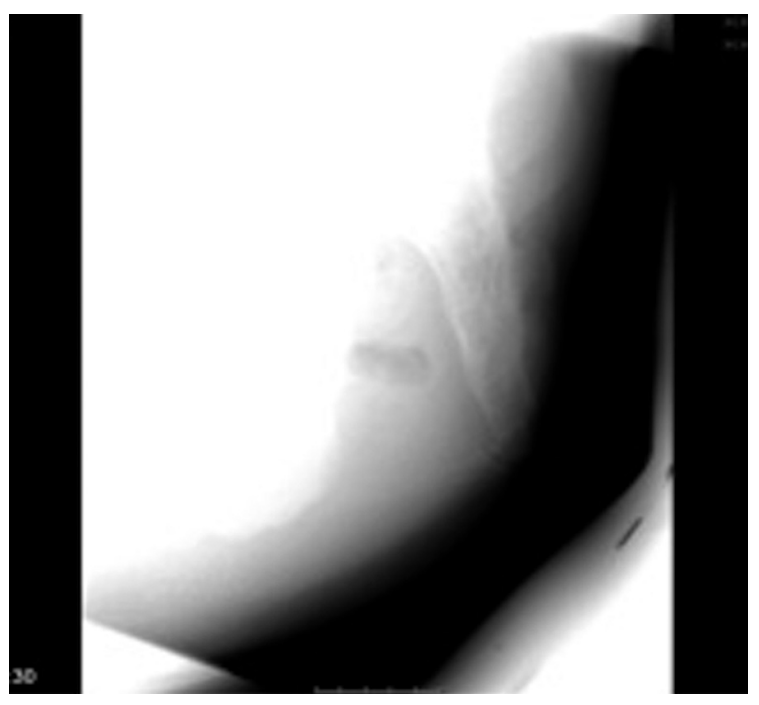

Fig. 1. Fracture of the coccyx on lateral X-ray view. 


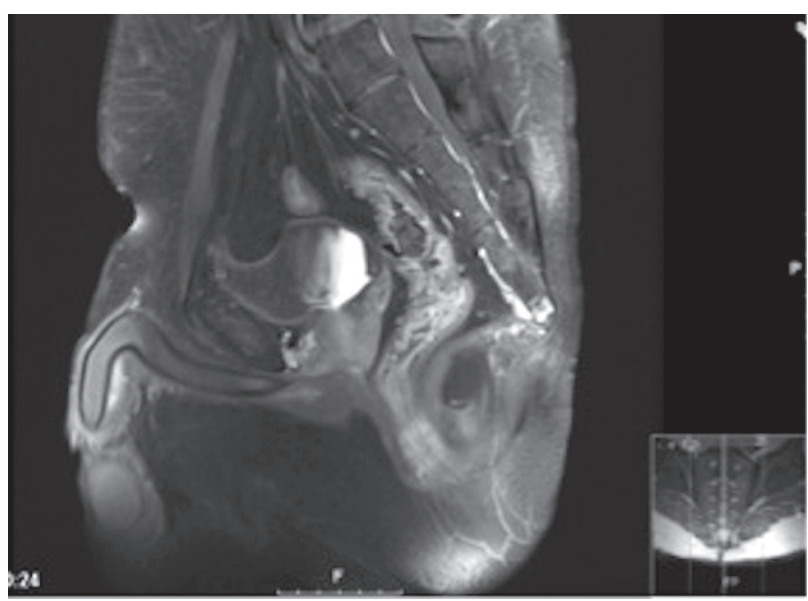

Fig. 2. View of coccyx-pseudarthrosis on lateral magnetic resonance imaging.

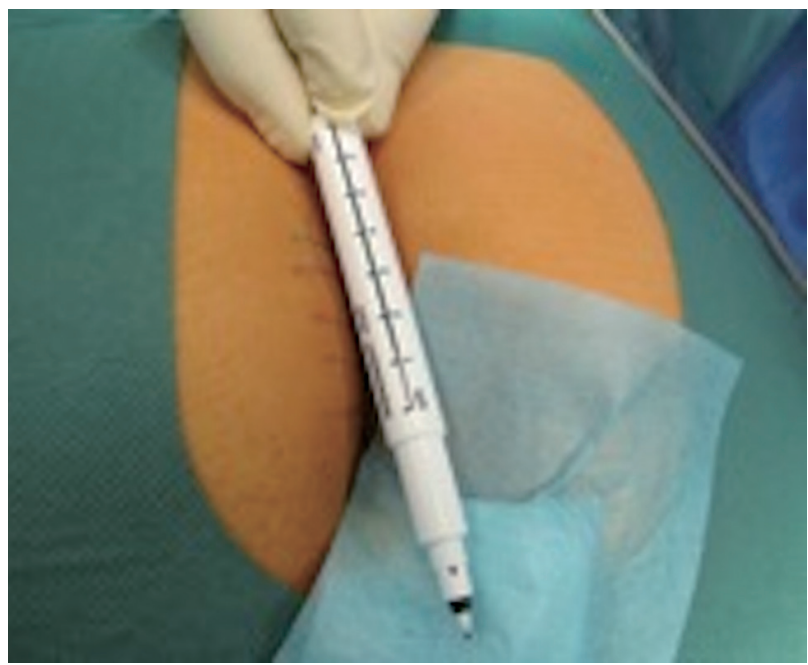

Fig. 3. Illustration of skin incision.

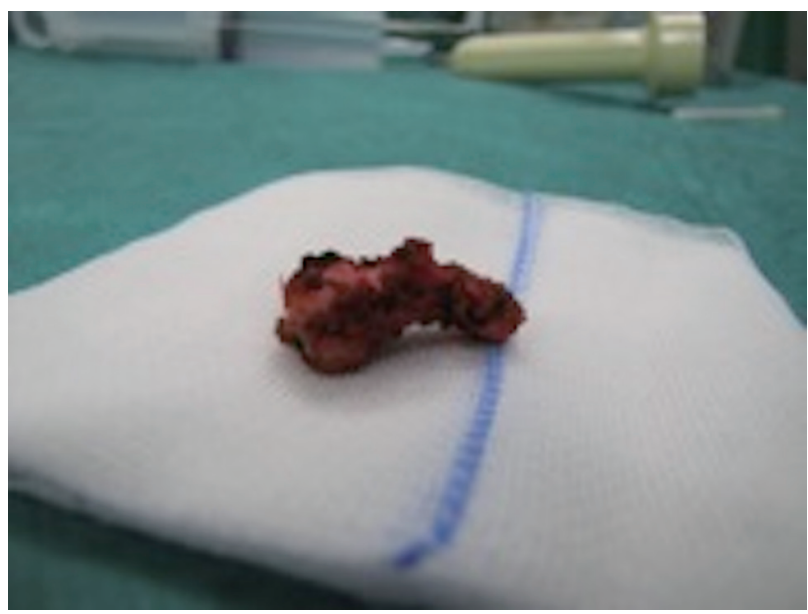

Fig. 4. Image of resected coccyx.
All patients participated in a follow-up examination as part of our postoperative clinical consultation. The patients were interviewed and examined at the one year postoperative mark in our outpatient clinic. The patients were asked to describe their current level of pain intensity using the visual analog scale (VAS). In addition, they were asked to estimate their general pain symptoms as one of five possible categories: complete pain relief, significant improvement of pain, moderate pain relief, unchanged pain and worsening of pain symptoms. Finally, the patients were asked whether their quality of life had improved after surgery, and whether they would advise this surgical intervention to a patient with similar symptoms. The success of the operation was determined on the basis of collected data. An "excellent" result was achieved with complete absence of pain or significant improvement of pain; this was operationalized as a VAS less than 2 of 10 and an increase in quality of life. A significant improvement in pain and a VAS less than 3 out of 10 was classified as a "good" result. A moderate improvement of pain and a VAS less than 6 out of 10 was classified as a "satisfactory" outcome. Unchanged pain symptoms, an increase in complaints, or a VAS at the time of the investigation of more than 6 out of 10 was classified as a "poor" result.

\section{Results}

All patients could be mobilized on the day of surgery. All patients were discharged from inpatient treatment with primary-wound healing and wounds that were free of inflammation. The average duration of hospital stay was 2.8 days.

One week after surgery, patient XY developed a woundhealing disorder with formation of a wound hematoma. This patient had experienced, however, reported falling onto the tailbone postoperatively. A reoperation was deemed necessary in this patient with wound revision and evacuation of the hematoma. There were no other reported complications during the hospitalization or the follow-up period. The results regarding the outcome were obtained one year postoperatively for all patients.

All patients benefited from surgery (as reflected in the data) and reported an overall improvement in their pain symptoms: 3 of the 10 patients had a complete absence of pain with an increase in their quality of life; an excellent result. Five patients reported a significant improvement in their pain symptoms with a VAS of less than 3 out of 10 
Table 1. Overview of the examined cases

\begin{tabular}{|c|c|c|c|c|c|}
\hline \multirow{2}{*}{ Case no. } & \multirow{2}{*}{ Age (yr)/gender } & \multicolumn{2}{|c|}{ Visual analog sacale } & \multirow{2}{*}{ Complication } & \multirow{2}{*}{ Outcomes } \\
\hline & & Preoperation & Postoperation & & \\
\hline 1 & 57/male & 8 & 2 & No & Excellent \\
\hline 2 & 36/male & 9 & 1 & No & Excellent \\
\hline 3 & 43/male & 8 & 1 & No & Excellent \\
\hline 4 & 44/male & 9 & 2 & $Y_{e s}{ }^{a)}$ & Excellent \\
\hline 5 & 42/male & 9 & 1 & No & Excellent \\
\hline 6 & 45/male & 10 & 2 & No & Excellent \\
\hline 7 & 46/female & 9 & 3 & No & Good \\
\hline 8 & 33/female & 10 & 3 & No & Good \\
\hline 9 & 35/female & 8 & 2 & No & Excellent \\
\hline 10 & 38/female & 8 & 2 & No & Excellent \\
\hline
\end{tabular}

alPostoperative wound hematoma after falling backside.

Table 2. Overview of compared relevant studies

\begin{tabular}{lccc} 
Study (author, year) & No. of cases & Excellent and good results (n, [\%]) & Complication rate (\%) \\
\hline Postacchini and Massobrio, 1983 [13] & 36 & $32(88)$ & 1.9 \\
\hline Wray et al., 1991 [14] & 23 & $21(91)$ & 0 \\
\hline Zayer, 1996 [10] & 10 & $10(100)$ & 10 \\
\hline Maigne et al., 2000 [4] & 37 & $34(92)$ & 30 \\
\hline Wood and Mehbod, 2004 [15] & 20 & $18(90)$ & 0 \\
\hline Cebesoy et al., 2007 [12] & 21 & $21(100)$ & 6.7 \\
\hline Sehirlioglu et al., 2007 [11] & 74 & $71(96)$ & 30 \\
\hline
\end{tabular}

and an increase in quality of life; also an excellent result. The remaining two patients reported a significant improvement of pain with a current VAS of 3 out of 10 and were classified as having achieved a good result. Therefore, $80 \%$ of the cases showed an excellent result and $20 \%$ of the cases showed a good result. Persistence or worsening of pain symptoms did not occur in any patient. In our study group, all patients suffered from posttraumatic coccygodynia with formation of pseudarthrosis. This was also confirmed intraoperatively, wherein visible instability of the coccyx to palpation was detected. All patients reported that they would repeat the procedure and would recommend it to patients with similar symptoms.

\section{Discussion}

All patients in this study achieved improvement in their pain/symptoms (by VAS) that reflected good or excellent results (Table 1). This correlates well with the results of similar studies in which more than $90 \%$ of the patients with traumatic coccygodynia also showed good or very good results after surgical coccygectomy (Table 2) [4,1015]. Several studies have indicated less benefit from surgery in patients with the idiopathic form of coccygodynia than in patients with a traumatic etiology $[12,16,17]$. However, other studies have shown no difference in patient outcomes between the traumatic and idiopathic forms of coccygodynia [13]. Because all patients in our series suffered from posttraumatic coccygodynia, a differentiation between the two types is not possible. Ruling out an associated psychological cause for the pain symptoms is of critical importance in patients with the idiopathic form of coccygodynia for whom the indications for surgery should be very strict $[14,18]$. 
Coccygectomy may seem to be a technically facile procedure. However, myriad complications have been documented in the extant literature. In a review of 24 studies involving 671 patients, an overall $11 \%$ complication rate was reported. Complication rates in studies varied from $0 \%$ [19] to 50\% [12]. The most common complications cited were: wound infections (8.34\%), and wound healing problems with dehiscence. Delayed healing $(0.9 \%)$ and wound hematomas $(0.3 \%)$ were also reported [20]. Wound infections were in the majority of cases superficial [20]. Serious complications, such as severe infections or injuries of the intestinal tract, have also been described; however, these were extremely rare [20]. In myriad studies, perioperative antibiotic prophylaxis was administered. The local skin flora, proximity to the anus, and hygiene difficulties due to the anatomical location of the operative site increases the risk of contamination. The most common causative agents of postoperative wound infection were gram-negative rods [17]. Several studies have shown that a five-day course of postoperative antibiotics (second-generation cephalosporins) can effectively reduce the infection rate $[4,9,12,15]$.

Patients with coccygodynia-especially if it is an idiopathic form-should be examined thoroughly to rule out any concomitant pathologies. Degenerative lumbar disease has been associated with coccygodynia $[5,13]$. Furthermore, colorectal disorders e.g., proctalgiafugax, rectal tumor, or descending perineum syndrome could mimic symptoms of coccygodynia [21,22]. In our series all patients suffered from traumatic coccygodynia with a clear traumatic event in their history.

Apart from total coccygectomy, a partial resection of the coccyx has also been described for the surgical treatment of coccygodynia. Postacchini reported no difference in the outcome for partial resection compared to that of complete coccygeal resection [13]. However, other authors have demonstrated superior postoperative outcomes with complete coccygectomy in comparison to partial [18] coccygectomy. In our series, only a complete resection was performed. A finding that has been reported in many studies, although it was not observed in our series, is the increased prevalence of the disease in females $[14,23]$. The reason for this finding is believed to be the particular anatomy of the female sacrum [14]. The coccyx is more prominent in women than in men and is thus more vulnerable to traumatic insults.

There remains still some skepticism in performing coc- cygectomy. Our study suggests that complete removal of the coccyx is a beneficial procedure and should be considered for chronic traumatic coccygodynia after conservative treatment has been exhausted. The authors acknowledge the weakness of this study as being a retrospective cohort study without a control group.

\section{Conclusions}

In summary, surgical coccygectomy in patients with trauma-induced coccygodynia is associated with good outcomes and can be justified as a therapeutic option after all conservative treatment options have been exhausted. Preoperatively, it is critical to explore possible co-morbidities or psychological factors and to strictly establish the corresponding indications for surgery. The patient must be informed about the increased risk of wound infection. Perioperative antibiotic prophylaxis and careful hygiene measures can significantly reduce this risk.

\section{Conflict of Interest}

No potential conflict of interest relevant to this article was reported.

\section{References}

1. Howorth B. The painful coccyx. Clin Orthop 1959; (14):145-60.

2. Duncan GA. Painful coccyx. Arch Surg 1937;34:1088104.

3. Johnson PH. Coccygodynia. J Ark Med Soc 1981; 77:421-4.

4. Maigne JY, Lagauche D, Doursounian L. Instability of the coccyx in coccydynia. J Bone Joint Surg Br 2000; 82:1038-41.

5. Fogel GR, Cunningham PY 3rd, Esses SI. Coccygodynia: evaluation and management. J Am Acad Orthop Surg 2004;12:49-54.

6. Patel R, Appannagari A, Whang PG. Coccydynia. Curr Rev Musculoskelet Med 2008;1:223-6.

7. Nathan ST, Fisher BE, Roberts CS. Coccydynia: a review of pathoanatomy, aetiology, treatment and outcome. J Bone Joint Surg Br 2010;92:1622-7.

8. Borgia CA. Coccydynia: its diagnosis and treatment. Mil Med 1964;129:335-8.

9. Hodges SD, Eck JC, Humphreys SC. A treatment and 
outcomes analysis of patients with coccydynia. Spine J 2004;4:138-40.

10. Zayer M. Coccygodynia. Ulster Med J 1996;65:58-60.

11. Sehirlioglu A, Ozturk C, Oguz E, Emre T, Bek D, Altinmakas M. Coccygectomy in the surgical treatment of traumatic coccygodynia. Injury 2007;38:1827.

12. Cebesoy O, Guclu B, Kose KC, Basarir K, Guner D, Us AK. Coccygectomy for coccygodynia: do we really have to wait? Injury 2007;38:1183-8.

13. Postacchini F, Massobrio M. Idiopathic coccygodynia. Analysis of fifty-one operative cases and a radiographic study of the normal coccyx. J Bone Joint Surg Am 1983;65:1116-24.

14. Wray CC, Easom S, Hoskinson J. Coccydynia. Aetiology and treatment. J Bone Joint Surg Br 1991;73:3358.

15. Wood KB, Mehbod AA. Operative treatment for coccygodynia. J Spinal Disord Tech 2004;17:511-5.

16. Pennekamp PH, Kraft CN, Stutz A, Wallny T, Schmitt O, Diedrich O. Coccygectomy for coccygodynia: does pathogenesis matter? J Trauma 2005;59:1414-9.
17. Bayne O, Bateman JE, Cameron HU. The influence of etiology on the results of coccygectomy. Clin Orthop Relat Res 1984;(190):266-72.

18. Hellberg S, Strange-Vognsen HH. Coccygodynia treated by resection of the coccyx. Acta Orthop Scand 1990;61:463-5.

19. Traub S, Glaser J, Manino B. Coccygectomy for the treatment of therapy-resistant coccygodynia. J Surg Orthop Adv 2009;18:147-9.

20. Karadimas EJ, Trypsiannis G, Giannoudis PV. Surgical treatment of coccygodynia: an analytic review of the literature. Eur Spine J 2011;20:698-705.

21. Mazza L, Formento E, Fonda G. Anorectal and perineal pain: new pathophysiological hypothesis. Tech Coloproctol 2004;8:77-83.

22. Parks AG, Porter NH, Hardcastle J. The syndrome of the descending perineum. Proc R Soc Med 1966;59: 477-82.

23. Tilscher H, Eder M. Spinal diseases: vertebral complaints in relation to site and age. Wien Med Wochenschr 1993;143:269-73. 